sind im Allgemeinen kleiner. Beiläufig ist zu bemerken, dass die Nichtübereinstimmung zwischen den Beobachtungen von 39 und 44 Bootis und der Correctionsformel ( $A-1$ ), die bei O. Struve sogar Zweifel über die Brauchbarkeit der letzteren erweckt hatte (Obs. de Poulkova IX, Introd. p. 108) durch die Einfuhhrung des Correctionsfactors $\sin z$ beseitigt wird.

Was die Beobachtungen vom Jahre 1839 bis 1843 betrifft, so bleibt es auch jetzt wegen der widersprechenden Resultate noch unentschieden, ob die periodischen Glieder der Formeln (A) oder (B) die Beobachtungen verbessern oder nicht.

O. Struve hat selbst bemerkt, dass seine systematischen Fehler sich mit der Zeit verändern. Um diese Veränderungen zu untersuchen, wurden alle vom Jahre 1839 bis 1890 gesammelten Beobachtungen in 10 Gruppen getheilt und, indem die in den Supplementen zu Band IX niedergelegten Coordinaten als Ausgangspunkt angenommen wurden, die folgenden mittleren Correctionen für jede Gruppe gebildet :

\begin{tabular}{rrrc} 
& & \multicolumn{2}{c}{ Mittlere Correction } \\
I & & für Abst. & für Pos. -W. \\
2 & $40-43$ & +0.034 & +0.8 \\
3 & $43-47$ & -0.012 & -0.7 \\
4 & $47-50$ & +0.031 & -1.1 \\
5 & $50-53$ & +0.029 & 0.0 \\
6 & $53-56$ & +0.057 & -0.1 \\
7 & $56-60$ & +0.008 & -0.4 \\
8 & $60-65$ & +0.011 & +0.3 \\
9 & $65-71$ & +0.011 & +1.1 \\
10 & $71-78$ & +0.014 & -0.7 \\
& $78-90$ & $(-0.02)$ & +0.6
\end{tabular}

Da O. Struve zu den Abständen der ersten Periode die Correction - o..08, zu denen der zweiten, dritten und vierten aber die Correction +o" 07 schon hinzugefügt hatte, so sind seine sämmtlichen constanten Correctionen für die verschiedenen Epochen folgende :

$$
\begin{array}{ll}
\text { I } & -0.046 \\
2 & +0.058 \\
3 & +0.101 \\
4 & +0.099 \\
5 & +0.057
\end{array}
$$

$$
\begin{array}{rr}
6 & +0.008 \\
7 & +0.011 \\
8 & +0.011 \\
9 & +0.014 \\
10 & (-0.02)
\end{array}
$$

Aus dem regelmässigen Gange dieser Correctionen kann man sich von ihrer Realität überzeugen, ohne ihre wahrscheinlichen Fehler zu berechnen, und eine leichte graphische Interpolation giebt ihre Werthe für jedes Jahr.

$\mathrm{Da}$ aber diese constanten Correctionen für die $\mathrm{Ab}$ stände sich mit den Abständen selbst verändern, so wurden alle Beobachtungen in drei Gruppen zusammengestellt :

\begin{tabular}{|c|c|c|c|c|}
\hline Jahr & $\begin{array}{c}I \\
0.4 \text { bis } I^{\prime \prime}\end{array}$ & $\begin{array}{c}\text { II } \\
I^{\prime \prime} \text { bis } 2^{\prime \prime}\end{array}$ & $\begin{array}{c}\text { III } \\
2^{\prime \prime} \text { bis } 4^{\prime \prime}\end{array}$ & \\
\hline 1844 & +0.04 & +0.04 & +0.07 & \\
\hline 1846 & +0.06 & +0.07 & +0.12 & \\
\hline 1848 & +0.07 & +0.08 & +0.14 & $-0.07 \quad(\mathrm{C}-1)$ \\
\hline 1850 & +0.08 & +0.09 & +0.15 & \\
\hline 1852 & +0.06 & +0.07 & +0.12 & \\
\hline 1854 & +0.04 & +0.04 & +0.08 & \\
\hline 1856 & +0.02 & +0.02 & +0.03 & \\
\hline 1858 & +0.01 & +0.01 & +0.01 & \\
\hline
\end{tabular}

Warschau, Sternwarte, 1898 October.

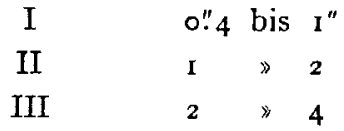

und es findet sich dann, dass die constanten Correctionen in diesen Gruppen sich im Verbältnisse von $0.74: 0: 8_{3}: 1.43$ vergrössern. Aus diesem Grunde wurden folgende constante Correctionen für Abstände abgeleitet, $z u$ welchen man vom Jahre 1843 bis $1853-0.07$ hinzufügen muss, wenn man die schon von Struve verbesserten Werthe der Beobachtungen annimmt.

Für die ubrigbleibenden constanten Correctionen der Positionswinkel, die gewöhnlich klein und von den Abständen ( 0.4 bis $4 " \circ$ ) unabhängig sind, erhält man, wenn man sie gruppenweise zusammenstellt, die folgenden Werthe :

$$
\begin{array}{ccc}
1843-1850 & -0.9 & \pm 0.19 \mathrm{w} . \mathrm{F} . \\
1850-1860 & -0.2 & \pm 0.13 \\
1860-1871 & +0.7 & \pm 0.17 \\
187 \mathrm{r}-1883 & -0.6 & \pm 0.19 \\
\text { nach } 1883 & (+1.0) & ( \pm 0.47)
\end{array}
$$

Diese Correctionen zeigen auch einen ziemlich regelmässigen Gang und können (mit Ausnahme der letzten) als reell betrachtet werden, denn sie geben dasselbe Maximum um das Jahr 1865 , welches von Thiele aus Struve's Beobachtungen der künstlichen Sterne gefunden worden ist (V.J.S. d. A. G. I5, S. 335 und folg.).

Da die Correctionen (C) aus den Beobachtungen selbst abgeleitet worden sind, so muss natürlich ihre Anwendung die oben erwähnten Quadratsummen verkleinern. Diese sind nach der Verbesserung der Beobachtungen nach den Formeln (B) und (C) folgende:

$$
\begin{aligned}
& \text { für Abstände } \quad 6.724 \\
& \text { » Positionswinkel } 99.57 \\
& \text { V. Ehrenfeucht }
\end{aligned}
$$

\title{
Die Perseiden von 1898.
}

Das aussergewöhnlich giunstige Wetter hat uns die Beobachtung einer ziemlich grossen Zahl von Perseiden ermöglicht, über die wir uns erlauben an dieser Stelle kurz zu berichten. Die Beobachtung geschah, ganz wie früher, unter Benutzung einer schwarzen Kugel, mit deren Hülfe für jede Nacht die Radianten hergeleitet wurden. -- Die Nächte von Aug. I I bis I 8 waren fast absolut klar, aber keineswegs vollständig dunkel; es fiel uns im Gegentheil neben der Durchsichtigkeit der Luft die Helligkeit des Himmels auf. Aug. 9 war die Heiterkeit etwa 0.7 bis 0.8 . Aug. 9 und i I hat der Mond (im Alter von 22 bezw. 24 Tagen) vielleicht die schwächeren Sternschnuppen unserer Beobachtung entzogen. 
In der Tabelle $I$ ist die Bedeutung der ersten fünf Spaiten an sich klar; die stündliche Zahl der Perseiden (sechste Spalte) ist, wo nöthig, auf die Heiterkeit r.o reducirt worden; auf den Mond wurde hierbei keine Rücksicht genommen; die letzte Spalte enthält das Tagesmittel der stündlichen Zahlen von Perseiden.

Tabelle I.



Aus der sechsten Spalte geht hervor, dass die Stunden vor und nach Mitternacht so ziemlich dieselbe Zahl von Perseiden lieferten. Bemerkenswerth ist die diesjährige sehr langsame Abnahme der Dichte des Schwarmes nach dem ro. August; man vergleiche A. N. 3401. Leider war am I o. August der Himmel fortwährend bewölkt, und es gestattete eine durch die Zahlen der siebenten Spalte bestimmte Curve nur annäherungsweise das Maximum des diesjährigen Perseidenfalles auf Aug. 10 $18^{\text {h }}$ zu ermitteln.
Die Tabelle II giebt eine Uebersicht der beobachteten Radianten; die zweite Spalte enthält das Datum der Be-


füntte die $Z$ ahl von Sternschnuppen, die mehr oder weniger genau den Radiationspunkt bestimmt haben.

Am I 7. und 18. August konnte kein Radiant aus den wenig zahlreichen Perseiden hergeleitet werden.

Tabelle II.

\begin{tabular}{|c|c|c|c|c|c|}
\hline \multirow{2}{*}{ Radiant } & \multirow{2}{*}{1898} & \multicolumn{2}{|c|}{1900.0} & \multirow{2}{*}{$\left|\begin{array}{l}\text { Zahl der } \\
\text { Perseid. }\end{array}\right|$} & \multirow{2}{*}{ Bemerkungen } \\
\hline & & $\alpha$ & $\delta$ & & \\
\hline I & & $48^{\circ}$ & $+5^{8^{\circ}}$ & 7 & \\
\hline II & Aug. $\quad 9$ & $5^{2}$ & $+5 I$ & 8 & \\
\hline III & & 353 & +62.5 & 3 & Unsicher \\
\hline IV & & $3^{2} \cdot 5$ & +35.5 & II & \\
\hline V & Aug. I I & $37 \cdot 5$ & $+5^{8}$ & 35 & \\
\hline VI & & 49 & +54 & 16 & \\
\hline VII & & 60 & +53 & 7 & \\
\hline VIII & & 18.5 & +50 & II & \\
\hline IX & Aug. 12 & 42 & $+5^{8}$ & 23 & \\
\hline$X$ & & 59 & +50 & 8 & \\
\hline
\end{tabular}

\begin{tabular}{|c|c|c|c|c|c|}
\hline \multirow{2}{*}{ Radiant } & \multirow{2}{*}{ I 898} & \multicolumn{2}{|c|}{1900.0} & \multirow{2}{*}{$\left|\begin{array}{l|}Z \text { ahl der } \\
\text { Perseid. }\end{array}\right|$} & \multirow{2}{*}{ Bemerkungen } \\
\hline & & $\alpha$ & 8 & & \\
\hline XI & & 3 & +60.5 & 4 & \\
\hline $\mathrm{XII}$ & & $37 \cdot 5$ & $+5^{8}$ & I 2 & $=\mathrm{V}$ \\
\hline XIII & & 44 & +53 & 1o & \\
\hline XIV & Aug. 13 & $5 I$ & +60.5 & I I & \\
\hline$X V$ & & 64 & +50 & 6 & \\
\hline XVI & & 77 & +83 & 4 & \\
\hline XVII & & 45 & +55 & 4 & $=$ XIII? Unsicher \\
\hline XVIII & Aug. I4 & 58 & +50 & 3 & $=\mathrm{X}$ ? Unsicher \\
\hline XIX & Aug. I 5 & 49 & +59 & 7 & $=$ XIV? \\
\hline $\mathrm{XX}$ & Aug. 16 & 60 & +59 & 8 & Gut \\
\hline
\end{tabular}

Bemerkungen zu der Tabelle II.

Aug. I 2. Bei der Bestimmung des Radianten IX ist Grösse beigelegt worden, welche um I $^{\mathrm{h}} 3^{\mathrm{m}} \mathrm{M} . \mathrm{Z}$. Utrecht grosses Gewicht einer fast stationären Sternschnuppe dritter aufleuchtete. 
Aug. 13. Auch heute wurde $\left(13^{h} 24^{m}\right.$ M. Z. Utrecht) eine fast stationäre Sternschnuppe (zweiter Grösse) beob. achtet, welche mit grossem Gewicht für die Ermittelung des Radianten XIV benutzt worden ist.

Aug. I 4. Auch der Radiant XVI war heute noch thätig. Aug. 15. Bei Polaris war ein zweiter Radiant thätig.

Wir ziehen es vor, vorläufig aus unseren eigenen Beobachtungen noch keine Schlüsse über Herrn Denning's seasterly motion of the radiante zu folgern. Zwar zeigt sich in den Radianten von etwa $59^{\circ} \mathrm{Decl}$. im Grossen und Ganzen

Utrecht, i 898 October 12. eine Verschiebung nach der Sonne hin, doch stimmen unsere Beobachtungen nicht mit Herrn Denning's Ephemeride (vgl. Knowledge i 898 S. 190).

Von 180 Perseiden bestimmten wir die Helligkeit, und zwar wurden $6,34,71,43,20$ Perseiden von der ersten, bezw. zweiten, dritten, vierten, fünften Grösse beobachtet, während dreimal die Helligkeit des Jupiter, dreimal sogar die der Venus beobachtet wurde. Die Farbe war im Durch. schnitt gelb; $z$ wei der hellsten Perseiden waren bestimmt orange, während deren sieben eine lebhaft grüne Farbe zeigten. Gekriimmte Bahnen haben wir nur vier gesehen.

Dr. A. A. Nijland, H. F. van Lummel.

\section{Ephemeris of the Radiant point of the Perseid meteoric shower.}

\section{By W.F. Denning.}

During a series of observations in August $1877 \mathrm{I}$ discovered that the Perseid radiant point exhibited a very definite motion, amounting to about $\mathrm{I}^{\circ}$ per day and directed to the East north east amongst the stars. In 1878 and subsequent years this feature was fully confirmed by a considerable number of additional observations and in the Monthly Notices, Vol. XLV p. 97, I gave a table shewing the varying strength of the shower and the place of its radiant during the 26 nights from July 25 to August 19. Fifteen years have elapsed since the publication of these results and $I$ have now revised and extended them on the basis of all my observations made at Bristol in the years from 1869 to 1898 and during the epoch comprising 40 days from July I I to August I 9. Within these dates I have observed more than 6479 meteors including about 2409 Perseids. In all I determined 73 Perseid radiant points and from these have prepared the following ephemeris which I believe will represent the place and motion of the shower very closely. I have included columns giving the average total horary number of meteors visible and also the horary rates of the Perseids and Aquarids during the same period. The position of the latter shower is at $339: 4-11 \% 6$, and it furnishes a rich display on the last few nights of July, as $I$ observed in 1878 . The radiant point appears to be persistent for a long interval of time.

Ephemeris of the Perseid Radiant point.

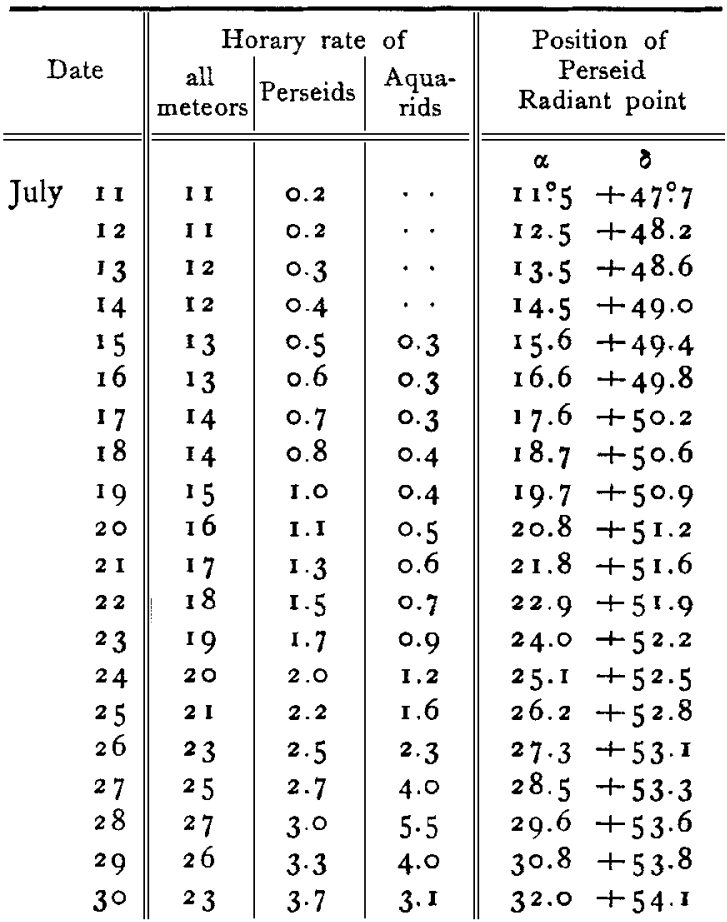

\begin{tabular}{|c|c|c|c|c|c|}
\hline Date & $\mid \begin{array}{c}\text { Ho } \\
\text { all } \\
\text { meteors }\end{array}$ & $\begin{array}{l}\text { orary rate } \\
\text { Perseids }\end{array}$ & $\begin{array}{l}\text { of } \\
\text { Aqua- } \\
\text { rids }\end{array}$ & \multicolumn{2}{|c|}{$\begin{array}{l}\text { Position of } \\
\text { Perseid } \\
\text { Radiant point }\end{array}$} \\
\hline July 3 I & 2 I & 4.1 & 2.2 & $\begin{array}{c}\alpha \\
33^{\circ}: 2\end{array}$ & $\begin{array}{c}8 \\
+54 \% 4\end{array}$ \\
\hline Aug. $\quad 1$ & 20 & 4.6 & 1.7 & 34.4 & +54.7 \\
\hline 2 & I 9 & 5.1 & 1.4 & $35 \cdot 5$ & +55.0 \\
\hline 3 & 19 & 5.7 & 1.2 & 36.7 & +55.2 \\
\hline 4 & $2 \mathrm{I}$ & 6.3 & I. $I$ & 37.9 & +55.5 \\
\hline 5 & 22 & 7.0 & 1.0 & 39.0 & +55.8 \\
\hline 6 & 24 & 7.9 & 0.9 & 40.2 & +56.0 \\
\hline 7 & 27 & 9.0 & 0.9 & $41 \cdot 4$ & $+5^{6.3}$ \\
\hline 8 & 34 & 15.0 & 0.8 & 42.6 & +56.5 \\
\hline 9 & 48 & 29.0 & 0.8 & 43.8 & +56.8 \\
\hline 10 & 69 & 50.0 & 0.7 & 45.0 & +57.0 \\
\hline I I & 48 & 29.0 & 0.7 & $4^{6} \cdot 3$ & +57.2 \\
\hline 12 & 30 & 12.5 & 0.6 & $47 \cdot 5$ & +57.5 \\
\hline 13 & 22 & 4.5 & 0.6 & 48.8 & +57.8 \\
\hline I 4 & 20 & 2.7 & 0.6 & 50.1 & +58.0 \\
\hline 15 & 18 & I. 5 & 0.5 & $51 \cdot 3$ & +58.2 \\
\hline 16 & 17 & 1.0 & 0.5 & 52.6 & $+5^{8.5}$ \\
\hline 17 & 16 & 0.7 & 0.5 & 53.9 & $+5^{8.7}$ \\
\hline I 8 & 15 & 0.5 & 0.4 & 55.2 & +58.9 \\
\hline 19 & 5 & 0.3 & 0.4 & 56.6 & +59.1 \\
\hline
\end{tabular}

Bristol, I 899 January II.

W. F. Denning. 